\title{
Site-selective depurination by a periodate-dependent deoxyribozyme $\uparrow$
}

\author{
Claudia Höbartner, P. I. Pradeepkumar and Scott K. Silverman*
}

\author{
Received (in Cambridge, UK) 26th March 2007, Accepted 3rd May 2007 \\ First published as an Advance Article on the web 10th May 2007 \\ DOI: $10.1039 / \mathrm{b} 704507 \mathrm{~g}$
}

\begin{abstract}
A deoxyribozyme is identified that mediates the site-selective depurination of its 5 -terminal guanosine nucleotide using periodate $\left(\mathrm{IO}_{4}{ }^{-}\right)$as an obligatory cofactor.
\end{abstract}

Nucleic acid enzymes (ribozymes and deoxyribozymes) that use small-molecule compounds as cofactors are interesting from both fundamental and applied viewpoints. The first report of a cofactordependent nucleic acid enzyme was of a deoxyribozyme that uses histidine to facilitate RNA cleavage. ${ }^{1}$ Oxidative cleavage of DNA by a deoxyribozyme can be achieved using $\mathrm{Cu}^{2+}$ either with or without ascorbate, ${ }^{2-4}$ and alcohol dehydrogenation or aldehyde reduction can be performed using $\mathrm{NAD}^{+}$or $\mathrm{NADH}$, respectively. ${ }^{5,6}$ A DNA aptamer for the fluorophore dihydrotetramethylrosamine has weak redox activity. ${ }^{7}$ The discovery that the natural RNA-cleaving $\operatorname{glm} S$ ribozyme requires glucosamine 6-phosphate (GlcN6P) as an obligatory coenzyme ${ }^{8,9}$ suggests that the use of cofactors by nucleic acid enzymes could have practical value. This is particularly important as efforts expand to apply RNA and DNA in many contexts, such as sensor applications. ${ }^{10,11}$

As part of our overall efforts to investigate the catalytic abilities of DNA, we designed a new in vitro selection experiment that was intended to use uridine $5^{\prime}$-diphospho- $N$-acetylglucosamine (UDPGlcNAc) as an electrophilic small-molecule substrate for attack by the DNA 5'-hydroxyl group as nucleophile, thereby attaching GlcNAc to the DNA. Separation of catalytically active deoxyribozymes during the selection procedure was to be achieved via a three-step procedure: (i) $\mathrm{NaIO}_{4}$ oxidation of the vicinal diol at positions 3 and 4 of the newly appended GlcNAc sugar ring; (ii) reductive amination with $\mathrm{NaCNBH}_{3}$ to attach a $3^{\prime}-\mathrm{NH}_{2}$ oligonucleotide; and (iii) PAGE separation of the deoxyribozyme sequences that were increased in length due to attachment of the $3^{\prime}-\mathrm{NH}_{2}$ oligonucleotide. $\$$ After 10 selection rounds, we were surprised to find that the $\mathrm{N}_{70}$ DNA pool had attained substantial catalytic activity (65-fold above background; Fig. 1A) that was independent of UDP-GlcNAc but required $\mathrm{NaIO}_{4}$. In particular, omission of periodate led to undetectable activity $(<0.5 \%)$. Furthermore, 5'-phosphorylation of the DNA pool suppressed the activity by at least 10-fold. $\S$ Apparently, periodate leads to formation of an amine-reactive moiety on the DNA, likely near the 5'-terminus, without first requiring attachment of GlcNAc that would be oxidized by the periodate. One individual deoxyribozyme, 10FN10, was chosen for further characterization of its periodate-dependent reaction.

Department of Chemistry, University of Illinois at Urbana-Champaign, 600 South Mathews Avenue, Urbana, Illinois 61801, USA.

E-mail: scott@scs.uiuc.edu; Fax: 217244 8024; Tel: 2172444489

$\dagger$ Electronic supplementary information (ESI) available: Details of all experimental procedures. See DOI: $10.1039 / \mathrm{b} 704507 \mathrm{~g}$

The 112-mer 10FN10 deoxyribozyme was incubated with $40 \mathrm{mM} \mathrm{NaIO}$ in the absence of UDP-GlcNAc in $50 \mathrm{mM}$ $\mathrm{NaOAc}\left(\mathrm{pH} \mathrm{5.2)}\right.$ at $37{ }^{\circ} \mathrm{C}$ for $2 \mathrm{~h}$. After precipitation to remove periodate, the DNA was subsequently treated with a 30-mer $3^{\prime}-\mathrm{NH}_{2}$ oligonucleotide and $10 \mathrm{mM} \mathrm{NaCNBH}$. PAGE revealed that $c a$. $10-20 \%$ of the DNA was converted to a slower-migrating band (Fig. 1B). This is consistent with periodate-induced formation of an amine-reactive functional group on the DNA and subsequent reductive amination with the added $3^{\prime}-\mathrm{NH}_{2}$ oligonucleotide. Treatment with $\mathrm{NaCNBH}_{3}$ during the latter step was required to observe the product. $\S$

We used strategically 2 -ribo-modified versions of $10 \mathrm{FN} 10$ to narrow the DNA reaction site by using internal transesterification at phosphorus ("alkaline hydrolysis") to follow the location of a ${ }^{32} \mathrm{P}$-radiolabel. Inclusion of a 2'-hydroxyl group at G16 of 10FN10 did not affect the reductive amination yield (not shown). The reductive amination product from rG16-10FN10 with a 15-mer $3^{\prime}-\mathrm{NH}_{2}$ oligonucleotide was $5^{\prime}-{ }^{32} \mathrm{P}$-radiolabeled, and subsequent cleavage by alkaline hydrolysis led to the expected product (Fig. 2A, rG16 lanes). Inclusion of 2'-hydroxyl groups at both G2 and G16 decreased the reductive amination yield by about 4-fold, but the rG2,rG16-10FN10 reductive amination product was still readily isolated by PAGE and $5^{\prime}-{ }^{32} \mathrm{P}$-radiolabeled. Cleavage of this product by alkaline hydrolysis showed the band expected if attachment of the $3^{\prime}-\mathrm{NH}_{2}$ oligonucleotide occurred at either the first or second nucleotide (G1 or G2; Fig. 2A, rG2,rG16 lanes). Importantly, attachment at the third nucleotide (A3) or further towards the 3 '-end of $10 \mathrm{FN} 10$ should lead to a larger cleavage band, which was not observed. Therefore, in the reductive

(A)

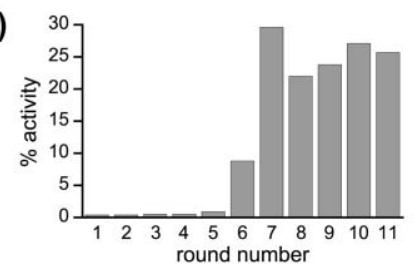

Fig. 1 Periodate-induced formation of an amine-reactive moiety by the 10FN10 deoxyribozyme. (A) Selection progression, shown as activity of the uncloned selection pool versus round number. A positive control substrate that bears a covalently attached GlcNAc moiety§ gave $26-44 \%$ yield when tested in parallel during each round. (B) Reaction of the periodate-treated round 10 DNA pool or the $10 \mathrm{FN} 10$ deoxyribozyme with a 30 -mer $3^{\prime}-\mathrm{NH}_{2}$ oligonucleotide and $\mathrm{NaCNBH}_{3}$ leads to $10-20 \%$ of the reductive amination product. The unreacted pool is shown as a standard. The slight gel shift between the standard and the other two lanes is from inclusion of a DNA splint that holds together the deoxyribozyme and the $3^{\prime}-\mathrm{NH}_{2}$ oligonucleotide (see supplementary information for reaction details) 


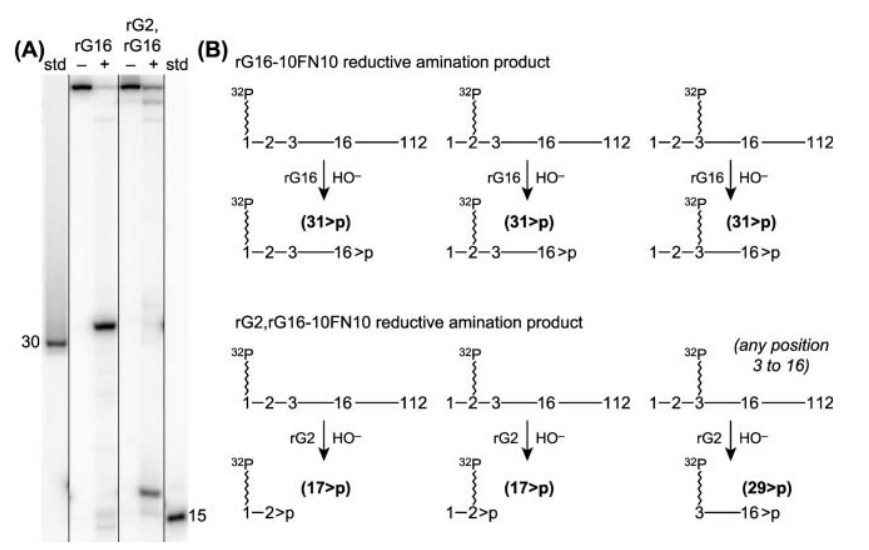

Fig. 2 Narrowing the site of reactivity to either G1 or G2 of the 10FN10 deoxyribozyme. (A) Gel image showing cleavage by alkaline hydrolysis (+) of the $5^{\prime}-{ }^{32} \mathrm{P}$-radiolabeled reductive amination product. $2^{\prime}$-Hydroxyl groups were incorporated into $10 \mathrm{FN} 10$ either at G16 (rG16) or at both G2 and G16 (rG2,rG16). Std = 15-mer or 30-mer 3'- NH $_{2}$ oligonucleotide. (B) Schematic depictions of the expected rG16-10FN10 and rG2,rG16$10 \mathrm{FN} 10$ cleavage products if the $15-\mathrm{mer}^{\prime}-\mathrm{NH}_{2}$ oligonucleotide (zigzag line) becomes attached at nucleotide G1, G2, or A3. For attachment at any of the first 16 positions, the control rG16-10FN10 cleavage product would be slightly larger than the 30-mer standard (i.e., 31-mer plus cyclic phosphate), as observed. Only in the case of attachment at G1 or G2 would the rG2,rG16-10FN10 cleavage product be slightly larger than the 15-mer standard (i.e., 17-mer plus cyclic phosphate), as observed. For attachment at A3 or any other position after G2, the rG2,rG16-10FN10 cleavage product would be a 29 -mer, which is not observed $(<0.5 \%)$.

amination product the $3^{\prime}-\mathrm{NH}_{2}$ oligonucleotide is attached at either G1 or G2.

We considered three likely candidates on the $10 \mathrm{FN} 10$ deoxyribozyme for the attachment site of the $3^{\prime}-\mathrm{NH}_{2}$ oligonucleotide by reductive amination (Scheme 1). First, the guanine nucleobase of $\mathrm{G} 1$ could be lost (i.e., depurination), followed by reductive amination of the $3^{\prime}-\mathrm{NH}_{2}$ oligonucleotide with the resulting abasic site (1). The initially unmasked aldehyde (1a) is also a 1,2-diol; therefore, 1a could be oxidized to a dialdehyde (1b). Second, the guanine nucleobase of G2 could be lost, leading to an abasic site that cannot be oxidized to a dialdehyde (2). Third, the 5'-hydroxyl group on nucleotide G1 of the DNA could be oxidized to a $5^{\prime}$-aldehyde (3). Sodium periodate was reported to oxidize benzylic alcohols under relatively harsh conditions, ${ }^{12}$ providing some precedent for this type of reaction (which would clearly require a catalyst to occur under mild conditions).

All three structures 1-3 are compatible with the outcomes of two additional experiments. $\S$ First, reduction of the $\mathrm{NaIO}_{4}$-treated product with $\mathrm{NaBH}_{4}$ before reductive amination entirely suppressed formation of the reductive amination product, consistent with direct reduction of an amine-reactive aldehyde functional group. Second, heating in $90 \mathrm{mM} \mathrm{NaOH}$ at $95{ }^{\circ} \mathrm{C}$ for $5 \mathrm{~min}$ after $\mathrm{NaIO}_{4}$ treatment entirely suppressed formation of the reductive amination product, consistent with strand scission (and therefore loss of the aldehyde functional group) induced by removal of a proton $\alpha$ to the carbonyl group. ${ }^{13}$ This latter experiment is also consistent with a fourth possibility for the oxidation product, a deoxyribonolactone, ${ }^{14}$ which could react with a $3^{\prime}-\mathrm{NH}_{2}$ oligonucleotide to form an amide. However, both the $\mathrm{NaCNBH}_{3}$ requirement and the suppression of product formation by
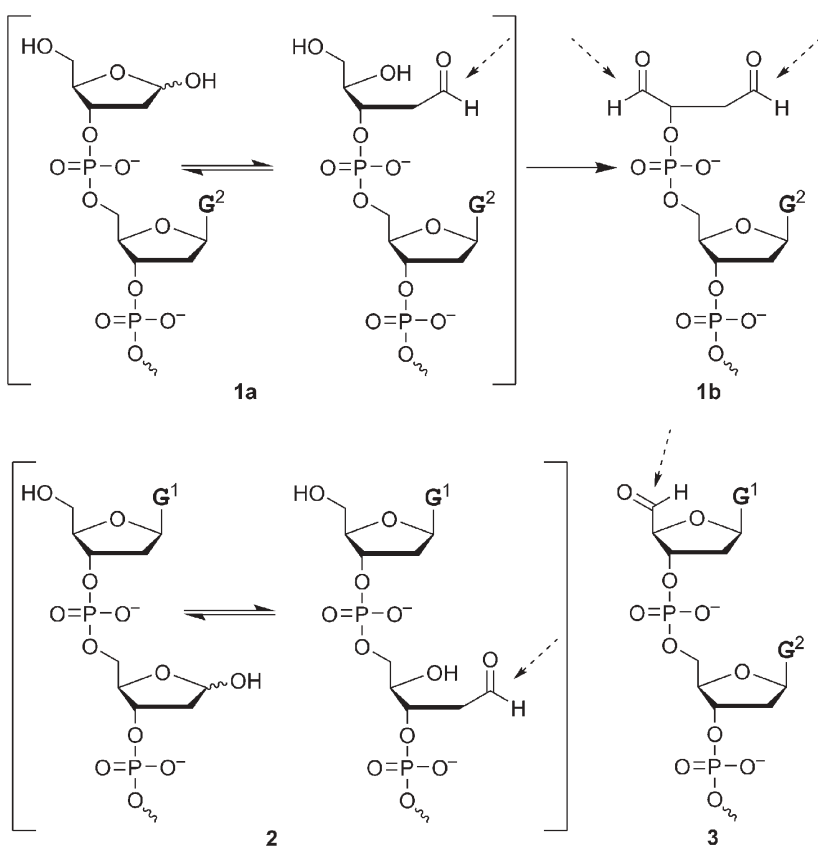

Scheme 1 Structural possibilities 1-3 for the product of reaction at nucleotide G1 or G2 of the 10FN10 deoxyribozyme before reductive amination. Compounds $\mathbf{1}$ and $\mathbf{2}$ represent depurination at G1 and G2, respectively, whereas 3 depicts oxidation of the 5 '-terminal hydroxyl group. Aldehyde functional groups are marked with dashed arrows, and guanine nucleobases are shown as G.

$\mathrm{NaBH}_{4}$ are inconsistent with the deoxyribonolactone structure, which should not require $\mathrm{NaCNBH}_{3}$ to form a stable adduct with a $3^{\prime}-\mathrm{NH}_{2}$ oligonucleotide and should not be reduced by $\mathrm{NaBH}_{4}$.

To determine more specifically which of $\mathbf{1}-\mathbf{3}$ corresponds to the periodate oxidation product, we performed the oxidation and reductive amination sequence using the $2^{\prime}$-ribo-G16 version of the 10FN10 deoxyribozyme (rG16-10FN10; $c f$. Fig. 2) and a 15-mer $3^{\prime}-\mathrm{NH}_{2}$ oligonucleotide. The product was PAGE-purified, and alkaline hydrolysis (i.e., cleavage at rG16) was used to remove the 3 '-terminal portion of the deoxyribozyme (this provides a smaller product and therefore more accurate mass determinations). Without further purification, analysis of the cleaved product by MALDI-MS revealed that the fragment containing both the $3^{\prime}-\mathrm{NH}_{2}$ oligonucleotide and the $5^{\prime}$-terminal portion of the deoxyribozyme has $\mathrm{m} / \mathrm{z} 9648 \pm 10$ (Fig. 3). $\uparrow$ The calculated $\mathrm{m} / \mathrm{z}$ values for the reductive amination products of $\mathbf{1 a}, \mathbf{1 b}, \mathbf{2}$, and $\mathbf{3}$ are 9691, 9643, 9691, and 9822, respectively (note that the $\mathrm{NaIO}_{4}$ oxidation $\mathbf{1 a} \longrightarrow \mathbf{1 b}$ removes two heavy atoms, as shown in Scheme 1, and the product from $\mathbf{3}$ would have much higher mass because it has not been depurinated). Of $\mathbf{1} \mathbf{- 3}$, the observed $\mathrm{m} / \mathrm{z}$ is consistent only with $\mathbf{1 b}$ as the structure of the product before reductive amination. The selectivity for formation of $\mathbf{1 b}$ over alternatives such as $\mathbf{2}$ or $\mathbf{3}$ can be conservatively estimated as $\geqslant 90 \%$, based on the lack of peaks at either higher expected $\mathrm{m} / \mathrm{z}$ value. Therefore, we conclude that $\mathrm{NaIO}_{4}$ induces site-selective depurination of the $10 \mathrm{FN} 10$ deoxyribozyme at nucleotide G1, leading to 1a. This subsequently forms $\mathbf{1 b}$ after periodate oxidation of the vicinal diol.

The mechanism of periodate-induced depurination at G1 is uncertain. Several additional experiments provide some clues. $\mathrm{KIO}_{4}$ is as effective a cofactor as $\mathrm{NaIO}_{4}$, but activity is undetectable $(<0.5 \%)$ if the nucleotide at position 1 is mutated 


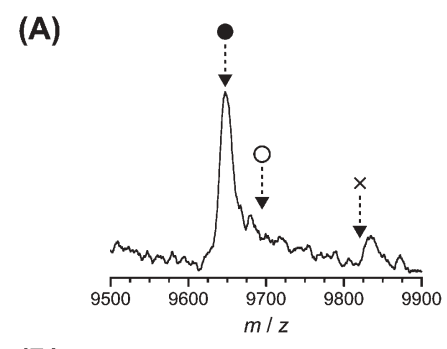

(B)

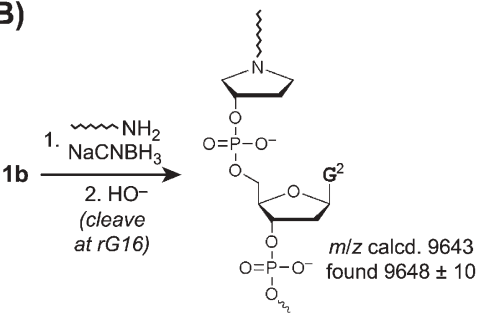

Fig. 3 MALDI mass spectrometry data showing that $\mathrm{NaIO}_{4}$ induces reaction of the 10FN10 deoxyribozyme by depurination of G1 to form 1a and subsequent oxidation to form $\mathbf{1 b}$. (A) Data trace obtained after alkaline cleavage of the reductive amination product from the rG16$10 \mathrm{FN} 10$ deoxyribozyme with the 15 -mer $3^{\prime}-\mathrm{NH}_{2}$ oligonucleotide. The filled circle marks the peak location expected if $\mathbf{1 b}$ is the final product of $\mathrm{NaIO}_{4}$ treatment; the open circle marks the location expected for either 1a or 2; and the X marks the location expected for 3. (B) Assigned structure of the observed reductive amination product from $\mathbf{1 b}$.

to anything other than guanosine.|| Modest activities ( 5-6-fold lower than with $\mathrm{NaIO}_{4}$ ) were observed with $40 \mathrm{mM} \mathrm{NaClO}$ or $1 \mathrm{mM} \mathrm{KMnO}_{4}$; this has not been investigated further. Oxidants such as $\mathrm{H}_{2} \mathrm{O}_{2}$ or ammonium persulfate led either to no observed reaction or to nonspecific DNA degradation, depending on concentration. Divalent metal ions such as $\mathrm{Mg}^{2+}, \mathrm{Ca}^{2+}, \mathrm{Zn}^{2+}$, $\mathrm{Co}^{2+}$, and $\mathrm{Cu}^{2+}$ (which were present during the original selection procedure) are not required for the reaction, which occurs with the same yield even in the presence of $5 \mathrm{mM}$ of the chelator EDTA.

Our data indicate that periodate is obligatory for the initial conversion of the 10FN10 DNA to 1a and not required solely for $\mathbf{1 a} \rightarrow \mathbf{1 b}$, which is a straightforward oxidation of a vicinal diol. If periodate were dispensable for formation of $\mathbf{1 a}$, then omission of periodate would lead to formation of the reductive amination product from 1a (instead of $\mathbf{1 b}$ ) ${ }^{13}$ but such a product is not observed in the absence of periodate. Moreover, periodate has not to our knowledge been reported to induce nonspecific oxidation or depurination of DNA, and indeed we do not observe any such nonspecific reactivity.§ One hypothesis for the role of periodate in the initial formation of 1a from $10 \mathrm{FN} 10$ is that the deoxyribozyme uses periodate as a cofactor to oxidize its $5^{\prime}$-terminal G1 nucleobase, ${ }^{15}$ and the oxidized nucleoside then spontaneously depurinates. An alternative hypothesis is that periodate oxidizes some other site on the deoxyribozyme, forming a nucleophile that catalyzes depurination in analogy to certain DNA repair enzymes. ${ }^{16,17}$ Because the mass spectrometry data are inconsistent with alteration of nucleotides G2 through G16 of the deoxyribozyme, any such oxidation must occur after position 16. Both of these hypotheses are reasonable, and further mechanistic investigations are required for their evaluation.

In summary, we have identified the $10 \mathrm{FN} 10$ deoxyribozyme that mediates site-selective depurination of its $5^{\prime}$-terminal guanosine nucleotide. The depurination reaction requires periodate as cofactor and does not involve divalent metal ions or $\mathrm{H}_{2} \mathrm{O}_{2}$. Cofactor-independent DNA depurination (deglycosylation) reactions have been reported ${ }^{18,19}$ but the requirement observed here for periodate indicates that $10 \mathrm{FN} 10$ leads to a different type of depurination reaction. The DNA-catalyzed reaction is site-selective because we do not detect any products arising from depurination at sites other than G1 (e.g., G2). In contrast, the $\mathrm{Cu}^{2+}$-dependent oxidative self-cleavage of several deoxyribozymes ${ }^{2-4}$ is not highly site-selective; indeed, the original report described those selfcleavage reactions as merely "region-specific". 4 Therefore, in several characteristics the 10FN10 deoxyribozyme provides a novel example of a cofactor-dependent DNA-catalyzed reaction.

This work was supported by the US National Institutes of Health (GM-65966 to S.K.S.) and an Erwin Schrödinger Fellowship from the Austrian Science Fund (to C.H.). We thank Marc Greenberg for comments on the manuscript and Shana Kelley for discussion of potential depurination mechanisms.

\section{Notes and references}

$\$$ The selection was performed using procedures similar to those in our previous report. ${ }^{20}$ See supplementary information for additional details of the selection procedure.

$\S$ See supplementary information for experimental details.

- See supplementary information for additional details of MALDI sample preparation and analysis.

\| Analysis of 10FN10 using the mfold secondary structure prediction algorithm did not suggest any strong secondary structure elements other than those involving the $5^{\prime}$-terminal nucleotides (see supplementary information). We have not performed any systematic experiments to investigate the secondary structure of $10 \mathrm{FN} 10$ in its $5^{\prime}$-terminal region.

1 A. Roth and R. R. Breaker, Proc. Natl. Acad. Sci. U. S. A., 1998, 95, 6027-6031.

2 N. Carmi, S. R. Balkhi and R. R. Breaker, Proc. Natl. Acad. Sci. U. S. A., 1998, 95, 2233-2237.

3 N. Carmi and R. R. Breaker, Bioorg. Med. Chem., 2001, 9, 2589-2600.

4 N. Carmi, L. A. Shultz and R. R. Breaker, Chem. Biol., 1996, 3, 1039-1046.

5 S. Tsukiji, S. B. Pattnaik and H. Suga, J. Am. Chem. Soc., 2004, 126, $5044-5045$.

6 S. Tsukiji, S. B. Pattnaik and H. Suga, Nat. Struct. Biol., 2003, 10, 713-717.

7 C. Wilson and J. W. Szostak, Chem. Biol., 1998, 5, 609-617.

8 T. J. McCarthy, M. A. Plog, S. A. Floy, J. A. Jansen, J. K. Soukup and G. A. Soukup, Chem. Biol., 2005, 12, 1221-1226.

9 J. C. Cochrane, S. V. Lipchock and S. A. Strobel, Chem. Biol., 2007, 14, 97-105.

10 R. Fiammengo and A. Jäschke, Curr. Opin. Biotechnol., 2005, 16, 614-621.

11 N. K. Navani and Y. Li, Curr. Opin. Chem. Biol., 2006, 10, 272-281.

12 T. M. Shaikh, L. Emmanuvel and A. Sudalai, J. Org. Chem., 2006, 71, 5043-5046.

13 J. Lhomme, J. F. Constant and M. Demeunynck, Biopolymers, 1999, 52 , 65-83.

14 L. Xue and M. M. Greenberg, Angew. Chem., Int. Ed., 2007, 46, 561-564.

15 S. Bjelland and E. Seeberg, Mutat. Res., 2003, 531, 37-80.

16 A. K. McCullough, M. L. Dodson and R. S. Lloyd, Annu. Rev. Biochem., 1999, 68, 255-285.

17 O. D. Schärer, Angew. Chem., Int. Ed., 2003, 42, 2946-2974.

18 T. L. Sheppard, P. Ordoukhanian and G. F. Joyce, Proc. Natl. Acad. Sci. U. S. A., 2000, 97, 7802-7807.

19 O. Amosova, R. Coulter and J. R. Fresco, Proc. Natl. Acad. Sci. U. S. A., 2006, 103, 4392-4397.

20 A. Flynn-Charlebois, Y. Wang, T. K. Prior, I. Rashid, K. A. Hoadley, R. L. Coppins, A. C. Wolf and S. K. Silverman, J. Am. Chem. Soc., 2003, 125, 2444-2454. 